\title{
Ultrafast Laser Structuring within a Process Chain - Influence of the Surface Morphology
}

\author{
Andreas Brenner, Martin Osbild and Johannes Finger \\ Fraunhofer Institute for Laser Technology (ILT), Steinbachstr. 15, Aachen, Germany, 52074 \\ E-mail: andreas.brenner@ilt.fraunhofer.de
}

\begin{abstract}
Ultrafast laser processing has shown its advantages characterized by a highly precise ablation with minimal thermal influence in many applications. Many investigations deal with the laser inherent parameters like pulse duration, repetition frequency and scan speed to describe their influence on the machining quality. However, the final machining quality is also determined by the initial surface morphology. To combine the conflicting objectives of productivity and quality a multi-level photonic process chain is established. For this kind of process chain, the knowledge about the influence of the respective surface morphology on the subsequent process step is of importance. For that reason the development of the surface morphology is investigated for several ultrafast laser parameter settings and initial surface morphologies. In our investigations, we were able to establish a mathematical model that describes the development of the roughness as a function of the ablation depth.
\end{abstract}

DOI: $10.2961 /$ jlmn.2019.03.0002

Keywords: ultrafast laser, micro structuring, surface morphology, process chain, quality, productivity, combination

\section{Introduction}

In the field of materials processing the production technologies are challenged by the two contradictory targets productivity and quality. Various efforts have been made in the past to address this conflict. The combination of different manufacturing processes are either performing parallel or sequential in which often one process is used to improve the quality achieved by the other.

Laser surface structuring as part of materials processing is becoming increasingly important in the industry for tool and mold making. While structured surfaces contribute to minimizing friction in combustion engines or to increasing efficiency of LED-based lighting systems due to their functionality, surface texturing is evolving a quality feature of products with regard to optical and haptic properties. Surface structuring by nanosecond pulsed laser sources is established for industrial use in a wide range of applications. A crucial factor for the success of the technology is to serve a high throughput. Ultrashort laser pulses still have shown their advantages regarding highly precise ablation while minimal thermal influencing the material in diverse applications. However, one of the main drawbacks is the low productivity of the ultrashort pulse laser process. [1-6]

One approach that combines the conflicting objectives of productivity and quality is to establish a sequentially photonic process chain for laser ablation. Motivated by mechanical roughing and finishing, a coarse ablation is carried out for example by means of a nanosecond laser, followed by fine ablation using a picosecond laser [7]. The ns process is extremely determined by melt dominated ablation that leads to high ablation rates. However, melt protrusions and debris accompany the process $[8,9]$. In contrast, by using ultrafast laser sources new design features with higher resolution can be fabricated. Due to the mainly vapor dominated ablation, highest precision can be achieved [10]. For this kind of photonic process chain, the knowledge about the influence of the respective surface morphology on the subsequent process step is of decisive importance.

Neuenschwander et al. investigated the influence of several different mechanical pretreatments of stainless steel and copper (sand blasting, grinding, polishing) on ultrashort pulsed laser ablation [11]. A brief impression how a laser pretreated surface (nanosecond structured) effects the surface finish using an ultrafast laser with pulse bursts gives Brenner et al. [12]. To sum up the essential findings of these both examinations there is a dependency of the final to the initial surface but still with a need for further investigation.

Preliminary investigations on grinded samples identified four different regimes of heat accumulation using ultrashort pulsed processing with burst respectively using high repetition frequencies (cf. Fig. 1) [12,13]. In the 1st regime, statistically distributed microstructures are formed at a burst energy of $<12 \mu \mathrm{J}$. There is almost no heat accumulation. Neuenschwander and Brenner showed a significant influence of the initial surface roughness on the final result by using ps parameter that can be assigned to this 1 st regime [11,12]. In the 2 nd regime, exactly the right amount of heat accumulation is achieved, resulting in a smooth surface with a homogeneous melt film. The more pulses per burst $(P p B)$ are used, the smaller the process window for this regime. A further increase in heat accumulation in the 3rd regime results in a stronger induction of melt. In this regime, the line distance and the bi-directionality of the scan strategy in the periodicity and orientation of the melt pearls become apparent. The surface structure is very regular. With a significant increase in heat accumulation in the 4th regime, the proportion of melt ablation increases decisively. Similar to ns ablation, this results in an irregular surface covered with melt ejection. 


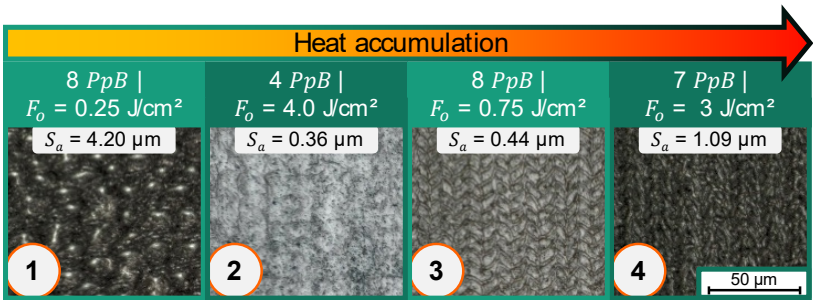

Fig. 1 Regimes of heat accumulation due to ultrashort pulsed burst processing.

These findings can be used for the main research activities presented in the underlying paper that explore the influence of the surface morphology after ns ablation to the subsequent ps ablation process. For that purpose initial grinded samples are structured by five different ns parameters that represent different surface quality levels. The final surface roughness depends on the applied ps ablation depth that is described by a mathematical model.

In the next section the methodology is described including the both used laser sources, the measuring equipment and the experimental design. The results are presented and discussed in section 3 . At first, the initial surfaces ablated by a ns process are analyzed and its influence on the surface quality and productivity of the subsequent ps ablation process is described. Afterwards, the relationship between the ablation depth and the surface roughness is investigated and mathematically described before finally an example of a process chain consisting of a combination of ns and ps ablation is shown.

\section{Method}

\subsection{Experimental setup}

The experimental tests are conducted on hot-working steel blanks (1.2738). The material samples have different initial quality levels. The basic condition of all samples is a grinded initial surface with an average surface roughness of $S_{a}=0.38 \pm 0.02 \mu \mathrm{m}$ and a roughness depth of $S_{z}=7.6 \pm 0.2 \mu \mathrm{m}$. The grinding grooves are straight and nondirectional. The grinded samples were treated by five different ns laser parameters that lead to different surface morphologies. To investigate the influence of these surface conditions on the further ps ablation process a broad range of ps laser parameters were applied producing squared cavities with a dimension of $5 \times 5 \mathrm{~mm}^{2}$ using a bidirectional scan strategy with an angle of $0^{\circ}$ and $90^{\circ}$. Detailed description of the different parameter settings is given in section 2.3.

The experimental setup consists of a 5-axis machining system AGIECHARMILLES LASER P 1000 U of GF MACHINING SOLUTIONS. The special feature of the system is that it has two laser sources with a common beam path. This allows combined ns/ps material ablation in a single workpiece clamping. For the ns processing a Q-switched IPG Ytterbium fiber laser $(\lambda=1064 \mathrm{~nm})$ is used with a pulse duration $\tau_{n s}$ of $150 \mathrm{~ns}$ and with a maximum average power of $P_{a v, n s}=100 \mathrm{~W}$. Its repetition frequency is variable between $5 \mathrm{kHz}$ and $200 \mathrm{kHz}$.

The ps processing is done by an ultrafast laser source from Edgewave with a maximum average power of $P_{a v, p s}=50 \mathrm{~W}$ and a pulse duration $\tau_{p s}$ of $10 \mathrm{ps}$. The amplifier is based on the InnoSlab concept, a design of the master oscillator power amplifier (MOPA) architecture that was developed at Fraunhofer ILT. The emitted laser radiation has a wavelength of $1064 \mathrm{~nm}\left(\mathrm{Nd}: \mathrm{YVO}_{4}\right)$ and an adjustable pulse repetition frequency $v_{\text {rep }}$ between $250 \mathrm{kHz}$ and $2 \mathrm{MHz}$. The seeder frequency $f_{\text {seed }}$ is $49.8 \mathrm{MHz}$. The laser beam is moved by a galvanometer scanner (IntelliSCAN III 14) and focused on the tool steel surface with a focal length of $f=125 \mathrm{~mm}$. The focus diameter $\left(1 / \mathrm{e}^{2}\right)$ is about $2 w_{0, n s}=40 \mu \mathrm{m}$ for the ns laser source and $2 w_{0, p s}=37 \mu \mathrm{m}$ for the ultrafast laser source (measured with a camera based beam profiler) at a theoretical Rayleigh length of about $z_{R, n s}=1.181 \mathrm{~mm}$ respectively $z_{R, p s}=1.011 \mathrm{~mm}$. To obtain reproducible and correct average overlapping pulses the feed per pulse (i.e. scan speed $v_{\text {scan }}$ ) and the line distance $(L D)$ is fixed at $8 \mu \mathrm{m}$ as well as the pulse repetition frequency with $500 \mathrm{kHz}$ for ps processing. For all applied ps laser ablation, the number of repeats $(N)$ is selected so that at the end of the ablation process the cavity has a depth of $z_{a b l}>30 \mu \mathrm{m}$ in order to keep the measurement error small compared to the overall ablation depth.

\subsection{Measuring equipment and target figures}

The central objectives of this work are the investigation of productivity and quality depending on the initial surface morphology. Productivity is quantified by the volume ablation rate $d V / d t\left[\mathrm{~mm}^{3} / \mathrm{min}\right]$, which can be determined as follows depending on the scan speed $v_{\text {scan }}$, the line distance $L D$, the ablation depth $z_{a b l}$ and the number of repeats $N$ :

$$
d V / d t=v_{\text {scan }} \cdot L D \cdot \frac{z_{a b l}}{N}
$$

Non-productive idle times such as deceleration and acceleration of the galvanometer scanner are not taken into account in this calculation. The ablation rate is averaged over the entire ablation process. In addition, the power specific volume ablation rate $d V / d t / P_{a v}$ for measuring the efficiency of the ablation process is determined as an auxiliary quantity.

The quality of the laser machined surfaces is quantified by the surface roughness $S_{a}[\mu \mathrm{m}]$. The $S_{a}$ value is calculated from a surface topography measurement and can be seen as an extension of the most common $R_{a}$ value by one dimension. In addition, the average roughness depth $S_{z}$ is measured as well. It is determined analog to $R_{z}$ from the absolute height difference between profile peaks and valleys.

The depth of ablation and the roughness are the two central measured variables of the experimental investigation. They are both determined using the KEYENCE VK-9700 Laser Scanning Microscope (LSM), which uses a semiconductor laser with a wavelength of $\lambda_{L}=408 \mathrm{~nm}$ for the topography measurement. The vertical resolution is about $0.5 \mu \mathrm{m}$ for measuring the depth and about $0.1 \mu \mathrm{m}$ for measuring the roughness. The lateral resolution of the $S_{a}$ images is about $0.43 \mu \mathrm{m}$.

The raw data are further processed by the software MOUNTAINSMAP from DIGITAL SURF. The determination of the ablation depth $z_{a b l}$ takes place via automatic step recognition, in which the height difference of two averaged planes is calculated. By using a Gaussian filter with different cut-offs, a wavelength dependent $S_{a}$ spectrum can be achieved as well as a global $S_{a}$ value without filtering. 
With the help of the spectrometer LAMBDA 1050 of the company PERKINELMER the absorption coefficient $A$ of the initial surfaces is determined. The measurement error is about $\pm 2 \%$. The visual evaluation of the experiments is supported by the KEYENCE VHX-5000 digital light microscope.

\subsection{Experimental design}

Fluence is known as the dominant influence on productivity and quality for ultrashort pulsed laser ablation. Since a central goal of the work is the increase of productivity, the fluence $F_{0}$ is varied beyond the efficiency maximum of the single pulse ablation $F_{0, \text { optimum }}=e^{2} \cdot F_{\text {thr }}$ [14-16] up to the maximum power of the laser source. Experimental investigations observed a threshold fluence of $F_{t h r}=0.11 \pm 0.01 \mathrm{~J} / \mathrm{cm}^{2}$ for the used steel blanks of 1.2738 and a pulse duration of $\tau_{p s}=10$ ps. $F_{0, \text { optimum }}$ than can be calculated to $0.81 \mathrm{~J} / \mathrm{cm}^{2}$. Besides the fluence the variation of the number of pulses per burst $(P p B)$ is of great interest due to their effects on the quality while increasing productivity. The energy expended increases linearly with the number of pulses per burst while the single pulse peak fluence remains constant.

\begin{tabular}{|c|c|c|c|c|c|c|c|c|c|}
\hline & \multicolumn{8}{|c|}{ Fluence $F_{0}\left[\mathrm{~J} / \mathrm{cm}^{2}\right]$} \\
\hline & & 1.00 & 1.25 & 1.50 & 2.00 & 2.50 & 3.00 & 3.50 & 4.00 \\
\hline \multirow{6}{*}{ 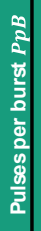 } & 3 & - & - & - & - & - & $36.2 \mu \mathrm{J}$ & $42.2 \mu \mathrm{J}$ & $48.3 \mu \mathrm{J}$ \\
\hline & 4 & - & - & - & $32.2 \mu \mathrm{J}$ & $40.2 \mu \mathrm{J}$ & - & - & $64.3 \mu \mathrm{J}$ \\
\hline & 5 & - & - & $30.2 \mu \mathrm{J}$ & $40.2 \mu \mathrm{J}$ & - & - & - & $80.4 \mu \mathrm{J}$ \\
\hline & 6 & - & $30.2 \mu \mathrm{J}$ & $36.2 \mu \mathrm{J}$ & $48.3 \mu \mathrm{J}$ & - & - & $84.4 \mu \mathrm{J}$ & - \\
\hline & 7 & $28.1 \mu \mathrm{J}$ & - & $42.2 \mu \mathrm{J}$ & - & - & $84.4 \mu \mathrm{J}$ & - & - \\
\hline & 8 & $32.2 \mu \mathrm{J}$ & $40.2 \mu \mathrm{J}$ & - & $64.3 \mu \mathrm{J}$ & $80.4 \mu \mathrm{J}$ & - & - & - \\
\hline
\end{tabular}

Fig. 2 Partial factorial experimental design with parameter variation of the ps ablation processed on ns pretreated surfaces.

For the investigation on the ns ablated initial surfaces, a compact, partial factorial experimental design is used that originates from the preliminary examinations mentioned in section 1. The parameter sets are shown in Fig. 2. Decisive for the selection are the expectation of a high surface quality, the coverage of a fluence spectrum as large as possible and a good comparability of the parameter sets with each other. Ablation with 1 and $2 P p B$ is not investigated because it tends to strong surface defects and does not have the necessary heat accumulation capacity to smooth the surface.

\begin{tabular}{|ccccccccc|}
\hline ISU & $\begin{array}{c}F_{0} \\
{\left[\mathrm{~J} / \mathrm{cm}^{2}\right]}\end{array}$ & $\begin{array}{c}v_{\text {scan }} \\
{[\mathrm{m} / \mathrm{s}]}\end{array}$ & $\begin{array}{c}L D \\
{[\mu \mathrm{m}]}\end{array}$ & $N$ & $\boldsymbol{A}[\%]$ & $\begin{array}{c}d V / d t \\
{\left[\mathrm{~mm}^{3} / \mathrm{min}\right]}\end{array}$ & $\begin{array}{c}S_{a} \\
{[\mu \mathrm{m}]}\end{array}$ & $\begin{array}{c}S_{Z} \\
{[\mu \mathrm{m}]}\end{array}$ \\
\hline $\mathrm{ns1}$ & 68.75 & 4.8 & 24 & 2 & 55.8 & 17.4 & 1.64 & 19.9 \\
$\mathrm{ns2}$ & 68.75 & 3.6 & 18 & 2 & 66.1 & 13.3 & 2.11 & 22.8 \\
$\mathrm{ns3}$ & 58.65 & 2.4 & 12 & 2 & 72.8 & 12.3 & 2.76 & 29.0 \\
$\mathrm{ns4}$ & 58.65 & 2.8 & 14 & 10 & 71.8 & 11.5 & 3.82 & 40.6 \\
$\mathrm{ns5}$ & 68.75 & 2.2 & 11 & 2 & 76.1 & 12.8 & 4.71 & 44.7 \\
\hline
\end{tabular}

Fig. 3 Nanosecond parameter that are applied to achieve five different ns pretreated initial surfaces with the corresponding absorption coefficient, ablation rate and surface roughness.

These ultrashort pulsed laser parameter sets are applied on five different ns pretreated initial surfaces. The used ns process parameter with the corresponding productivity and surface quality are shown in Fig. 3. In the following, the term "initial surface" is abbreviated as "ISU".
All ablated cavities and all laser pretreated initial surfaces are cleaned with a ten percent hydrochloric acid solution before measuring or further ps ablation.

\subsection{Initial surfaces ablated by nanoseconds}

The ns ablated initial surfaces differ from surfaces ablated by ps, in particular in a larger surface roughness of up to $S_{a}=4.7 \mu \mathrm{m}$ and the existence of a pronounced heat-affected zone. The five ns ablated initial surfaces ns1 to ns5 hardly differ from each other in structure because of the undefined, irregular shape of solidified melt, as shown in Fig. 4. Only the depth of the structure is different.

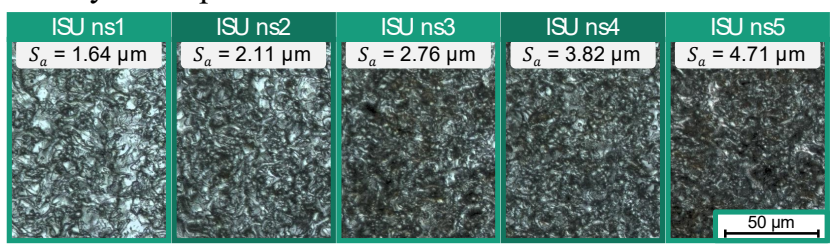

Fig. 4 Microscopic images of the ns pretreated initial surfaces.

From the measured absorption spectra, only the absorption coefficient at the laser wavelength $\lambda_{L}=1064 \mathrm{~nm}$ is relevant for this work. The absorption coefficient of the initial grinded steel blank is about $40 \%$. For the ns ablated ISU ns 1 $\left(S_{a}=1.64 \mu \mathrm{m}\right)$ an increased absorption coefficient can be measured with $55.8 \%$. A correlation to the roughness can be seen for the ns ablated initial surfaces. The roughest ISU ns5 $\left(S_{a}=4.71 \mu \mathrm{m}\right)$ achieves an absorption coefficient of $A=76.1 \%$. The absorption coefficients of the other initial surfaces are in-between.

The following statements on the absorption coefficient only apply to the first ablated layer done by the ps laser source. At each further repeat, the laser beam encounters an already modified surface with altered absorption properties. The quantitative data do not take into account the change in the degree of absorption during a pulse, which depends on the pulse duration and mainly results from a briefly increased electron temperature. [17]

\section{Results and Discussion}

\subsection{Influence of initial surface on achieved surface quality}

The analysis of this test series illustrates the significant influence of the initial surface on the quality of the ablation result. The exactly same ps parameters produce a final surface quality that correlates with the initial roughness of the different ns ablated initial surfaces. At $3 P p B$ there is a limit for a maximum roughness that can still be machined, as can be clearly seen in Fig. 5 .

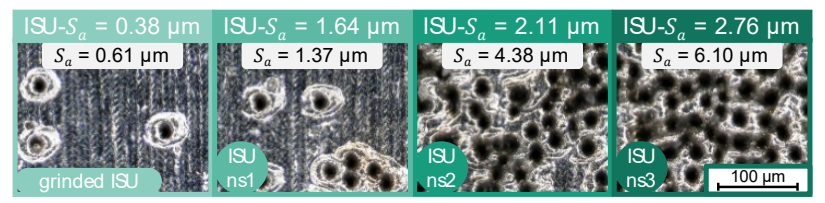

Fig. 5 Microscopic images showing the influence of the initial surface on the final surface morphology applying $3 \boldsymbol{P p} \boldsymbol{B}$ with $\boldsymbol{F}_{\mathbf{0}}=4.0 \mathrm{~J} / \mathrm{cm}^{2}$

Applying $3 P p B$ with $F_{0}=4.0 \mathrm{~J} / \mathrm{cm}^{2}$ tends to build pits already on the initially grinded ISU. But the averaged surface roughness only slightly drops from $0.38 \mu \mathrm{m}$ to $0.61 \mu \mathrm{m}$. 
The ablation on the ISU ns1 causes a reduction of the roughness from original $1.64 \mu \mathrm{m}$ to $1.37 \mu \mathrm{m}$. However, if the initial roughness is higher, as it is the case for ns 2 and ns 3 , the already rough ISU becomes even rougher due to extreme crater formation. There will be no improvement in surface quality with an increasing number of repeats.

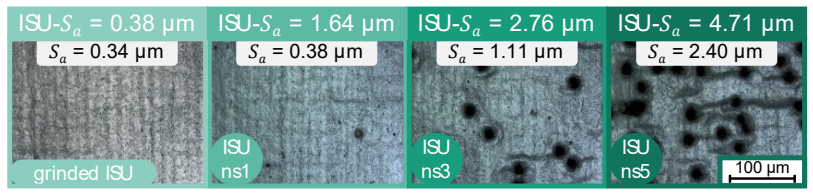

Fig. 6 Microscopic images showing the influence of the initial surface on the final surface morphology applying $4 \boldsymbol{P p B}$ with $\boldsymbol{F}_{\mathbf{0}}=2.5 \mathrm{~J} / \mathrm{cm}^{2}$.

In contrast to a burst with 3 pulses, 4 pulses within a burst ensure a stronger development of melt that leads to a noticeable smoothing of the initial roughness. When looking at Fig. 6, the described effect becomes obviously. As the initial roughness increases, so does the number of pits on the otherwise smooth surface covered by a thin layer of melt. At this point, the use of the roughness depth $S_{z}$ is indispensable. The $S_{z}$ values for ISU ns3 and ns5 are $29.0 \mu \mathrm{m}$ and $44.7 \mu \mathrm{m}$, respectively. However, the respective ablation depth is only $z_{a b l}=33.9 \mu \mathrm{m}$ and $z_{a b l}=30.9 \mu \mathrm{m}$ for the parameter set under consideration with $4 P p B$ and $F_{0}=2.5 \mathrm{~J} / \mathrm{cm}^{2}$. For a successful smoothing of the surface, a minimum ablation depth is required, which is significantly higher than the $S_{z}$ value of the ISU. The smoothing mechanism is based on the combination of two process variants. On the one hand, the smoothing is carried out by means of ablation, in which the profile peaks are removed more than the rest of the surface. On the other hand, parallel to the ablation process the material is remelted. Due to the interaction of these both smoothing mechanisms, the roughness can be further reduced and pits can be eliminated by increasing the ablation depth. In section 3.3.2, a mathematical relationship between the ablation depth $z_{a b l}$ and the roughness depth $S_{z}$ is established.

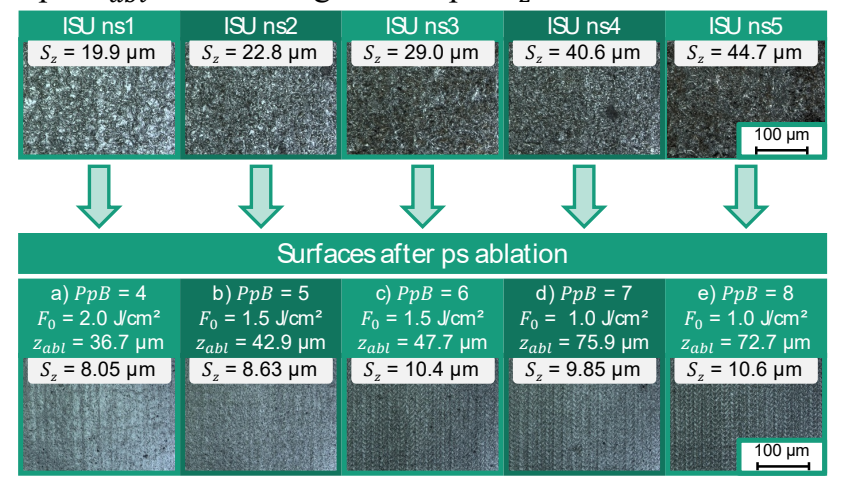

Fig. 7 Change of the surface morphology of each ns pretreated initial surface by successful ablation and smoothing with different ps burst parameters.

The ablation tests with $P p B \geq 5$ are accompanied by a potentially sufficient heat input for smoothing. For all ns ablated initial surfaces, the initial roughness is reduced to a roughness of $S_{a}<1 \mu \mathrm{m}$ within the ablation depth. Purely visually no difference can be determined to the reference cavities ablated on the grinded ISU. In general, it can also be concluded that by an increasing number of $P p B$ the sensitiv- ity to the ISU morphology decreases - regardless of the fluence used $\left(F_{0}=1.0-4.0 \mathrm{~J} / \mathrm{cm}^{2}\right)$ and under the condition of a sufficient ablation depth. Example images for a successful smoothing of the ns ablated initial surfaces are shown in Fig. 7.

The ablation quality as a function of the original surface cannot be generalized in a trivial way. The following hypothesis is derived on the basis of regularities observed: In ultrashort pulsed laser ablation with bursts, a smoothing ability can be determined for each process parameter combination. The extent of the smoothing potential is determined by the heat input due to heat accumulation that is dependent on the process parameters fluence $F_{0}$, pulse overlap $P O$, repetition frequency $f_{\text {rep }}$, seed frequency $f_{\text {seed }}$ and in particular the number of pulses per burst $P p B$.

Each process parameter combination with a specific smoothing ability is linked to a threshold roughness depth $S_{z, t h r}$. If the initial roughness depth of the ISU $S_{z, I S U}$ is below this threshold $\left(S_{z, I S U}<S_{z, t h r}\right)$, the roughness of the ISU can still be positively influenced by ps ablation. The roughness depth converges then with rising ablation depth $z_{a b l}$ against an asymptotic saturation roughness depth $S_{z, \infty}$, which depends only on the process parameters and not on the condition of the initial surface:

$$
\lim _{z_{a b l} \rightarrow \infty}\left(S_{z}\left(z_{a b l}\right)\right)=S_{z, \infty} \text { for } S_{z, I S U}<S_{z, t h r}
$$

In the case that the roughness depth of the ISU exceeds the threshold roughness depth corresponding to the respective smoothing capacity $\left(S_{Z, I S U}>S_{Z, t h r}\right)$, no smoothing to $S_{z, \infty}$ can be caused. Instead, a high roughness is intensified by any subsequent ablation process. In the worst case, a too rough ISU can also be a breeding ground for the production of extremely rough, self-organized microstructures (CLP). These surfaces are many times rougher than the initial surface, as can be seen from the example of $3 P p B$ (c.f. Fig. 5). The development of these microstructures also leads to a significant drop in productivity. The formation of CLPs is not completely understood and subject of current research activities.

However, the model is not universally applicable to burst processing. Parameters that fit to the 1st regime of heat accumulation (e.g. $8 P p B$ and $F_{0}=0.25 \mathrm{~J} / \mathrm{cm}^{2}$ ) have a worse smoothing ability that lead to self-organized structures even on grinded initial surfaces. Parameters without any heat accumulation do a better job than those with a slightly melt production in that case. In the context of this work, the observations for heat accumulation of the 2 nd to 4 th regime agree very well with the developed model without any exception. In section 3.3.2 a mathematical model is established that describes the development of the roughness as a function of the ablation depth.

\subsection{Influence of initial surface on productivity}

On all laser-pretreated initial surfaces a higher productivity of the ps ablation can be observed in comparison to reference ablation on the grinded initial surface. This observation must not be taken as a general result of the advanced incubation effect. The incubation effect indicates an irreversible lowering of the ablation threshold, which has a productivity-increasing effect. In the studies published so far, 
however, only the number of repeats was varied, while all other process parameters remained constant. [13,18-20] In contrast, in this study the same surface is treated with completely different parameter sets and in particular with different pulse durations, which cause different ablation mechanisms. Therefore, the discussion about the increase in productivity must be more differenced. The productivity of a subsequent ps ablation process is significant influenced by the ns pretreated surfaces. The findings can be summarized qualitatively as follows:

- Applying $\geq 5 P p B$ leads to an increased ablation rate (=productivity) compared to reference examinations on a grinded initial surface.

- The development of crater-shaped microstructures on very rough initial surfaces causes strong losses in the ablation rate. A decrease of the ablation rate by $35 \%$ is observed e.g. for $3 P p B$ and $F_{0}=4.0 \mathrm{~J} / \mathrm{cm}^{2}$ at $N=44$ on the roughest ns ablated ISU (ns5) compared to ablation on the grinded surface. For $P p B \geq 5$, these microstructures do not occur even on the roughest ISU, so the ablation rate is not negatively affected.

- The ablation rate tends to increase with the roughness of the initial surface. Besides other effects this behavior is due to the degree of absorption, which correlates with the roughness.

- The increase of the ablation rate seems to have a saturation. The ablation rate on the ns ablated initial surfaces with $S_{a}>2.7 \mu \mathrm{m}$ (ns3, ns4 \& ns5) does not differ significantly from each other.

The main reason for the higher ablation rate on the laser pretreated surfaces seems to be the measured higher absorption coefficient. There is a correlation to the greater roughness depth of the laser-ablated initial surfaces. The higher the roughness depth $S_{z}$, the more repeats are needed until the roughness is gradually smoothed. This means that the increased roughness depth is maintained over a greater ablation depth. Thus, the increased absorption also exists over a greater ablation depth, which is why the increase in the ablation rate correlates with the initial roughness. A lower initial roughness depth is "overwritten" after a few repeats. In that cases the parameter specific saturation roughness $S_{z, \infty}$ is reached, that shows the same degree of absorption like the grinded surface.

Any laser pretreatment achieves an increase in the ablation rate for the subsequent ps ablation. Since the presence of an oxide layer can also be excluded due to cleaning, laserinduced stresses in the interior of the workpiece can be assumed, which reduce the threshold fluence.

\subsection{Influence of the ablation depth on the achieved sur- face morphology}

Due to equation (1) the dependence of the final surface roughness on the ablation depth is obvious. In order to investigate the quantifiable influence of the ablation depth for ps ablation a step by step analysis after several repeats is done. The initial surface ns5 serves as an extreme case with its surface roughness $S_{a}=4.71 \mu \mathrm{m}$ and its roughness depth of $S_{z}=44.7 \mu \mathrm{m}$.

\subsubsection{Development of the surface roughness with in- creasing ablation depth}

The experimental parameters are presented in Fig. 8. The application of both parameters shows a very similar ablation behavior, which is why in the following only the analysis of parameter set 1 will be presented as representative for both parameters. The grinded sample with an initial surface roughness of $S_{a}=0.38 \mu \mathrm{m}$ is pretreated by ns laser ablation resulting in a surface roughness of $S_{a}=4.71 \mu \mathrm{m}$.

\begin{tabular}{|lcc|}
\hline & ps parameter set 1 & ps parameter set 2 \\
\hline Pulses per burst $P p B$ & 6 & 8 \\
Fuence $F_{0}$ & $1.5 \mathrm{Jcm}^{2}$ & $1.0 \mathrm{Jcm}^{2}$ \\
Number of repeats $N$ & \multicolumn{2}{c}{$10-250$} \\
Pretreatment ISU & \multicolumn{2}{c}{ ISU ns5 } \\
Roughness ISU $S_{a}$ & $4.7 \pm 0.5 \mu \mathrm{m}$ \\
Roughness depth ISU $S_{z}$ & \multicolumn{2}{c}{$48.0 \pm 3.7 \mu \mathrm{m}$} \\
\hline
\end{tabular}

Fig. 8 Picosecond parameter for the investigation of the development of the surface roughness depending on the ablation depth.

Initially, the surface roughness decreases very quickly with an increasing ablation depth. For an ablation depth $>60 \mu \mathrm{m}$ there is no significant improvement in surface quality recognized. The Fig. 9 shows the development of the surface roughness $S_{a}$ and the surface morphology in respect to the number of repeats $N$ treated by picosecond laser ablation using pulse bursts.

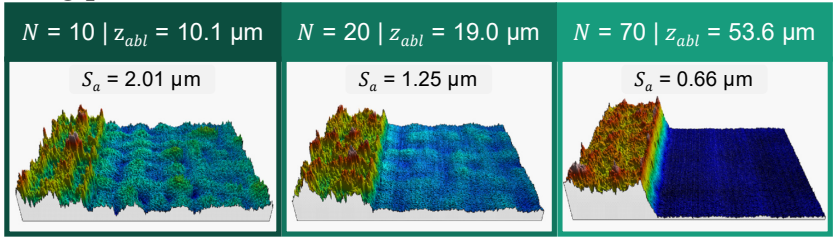

Fig. 9 Evolution of the surface morphology depending on the number of repeats $N$ (respectively ablation depth $\boldsymbol{z}_{\boldsymbol{a} \boldsymbol{b l}}$ ) applying $6 \boldsymbol{P} \boldsymbol{p} \boldsymbol{B}$ and $\boldsymbol{F}_{\mathbf{0}}=1.5 \mathrm{~J} / \mathrm{cm}^{2}$ on the ns ablated initial surface ISU ns5 with $\boldsymbol{S}_{\boldsymbol{a}}=4.71 \mu \mathrm{m}\left(\boldsymbol{S}_{\boldsymbol{z}}=44.7 \mu \mathrm{m}\right)$.

The analysis of the $S_{a}$ spectrum can be done in a detailed manner by considering the spatial wavelength. It describes the lateral distance between two consecutive peaks. By using a cut-off filter the surface roughness of a specific spatial wavelength regime can be analyzed. Depending on the spatial wavelength the surface can be divided in the regimes micro roughness $(\lambda<10 \mu \mathrm{m})$, meso roughness $(10 \mu \mathrm{m}<\lambda<80 \mu \mathrm{m})$ and macro roughness $(\lambda>80 \mu \mathrm{m})$. Plotting the $S_{a}$ spectrum as a function of the number of repeats (cf. Fig. 10) shows that the roughness is reduced across all spatial wavelength ranges (global $S_{a} / S_{z}$ ). Already after 20 repeats, which in this experiment corresponds to an ablation depth of $z_{a b l}=19.0 \mu \mathrm{m}$, the surface reaches its minimum possible roughness in the local wavelength range $\lambda<20 \mu \mathrm{m}$. Further repeats lower only the roughness in the local wavelength range $\lambda>20 \mu \mathrm{m}$.

For this area, the majority of the roughness decrease can presumably be attributed to the principle of smoothing by ablation (cf. section 3.1), since smoothing by remelting cannot take place at such large spatial expansions if the focus diameter is only $37 \mu \mathrm{m}$. As soon as the roughness in this spatial wavelength range $(\lambda>20 \mu \mathrm{m})$ is below the micro roughness, no further change in the global $S_{a}$ or $S_{z}$ value is noticeable. 


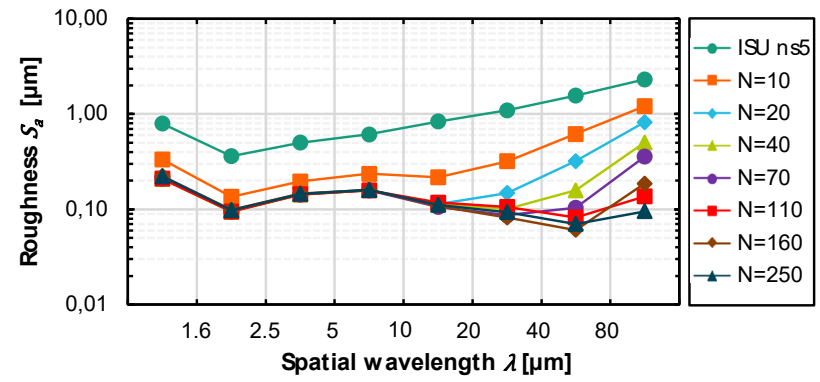

Fig. 10 Development of the $\boldsymbol{S}_{\boldsymbol{a}}$ spectrum over the spatial wavelength as a function of the number of repeats $\boldsymbol{N}$ for $6 \boldsymbol{P p B}$ and $\boldsymbol{F}_{\mathbf{0}}=1.5 \mathrm{~J} / \mathrm{cm}^{2}$ on the ns ablated initial surface ISU ns5 with $\boldsymbol{S}_{\boldsymbol{a}}=4.71 \mu \mathrm{m}\left(\boldsymbol{S}_{\boldsymbol{z}}=44.7 \mu \mathrm{m}\right)$.

\subsubsection{Theoretical approach for roughness depth as a function of ablation depth}

The idea of the roughness depth, which decreases with increasing ablation depth, is illustrated in Fig. 11. The figure summarizes the mechanisms of action that lead to a decrease of surface roughness while material is ablated and remelted simultaneously.

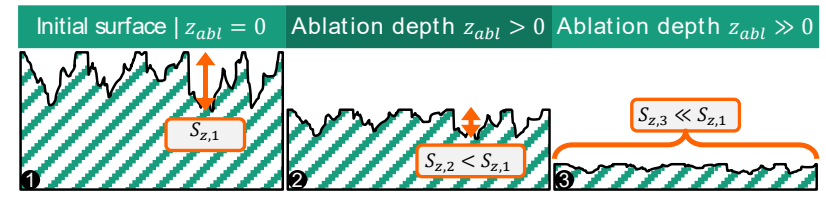

Fig. 11 Schematic diagram of the decreasing roughness depth $\boldsymbol{S}_{\boldsymbol{Z}}$ as a function of the ablation depth $\mathbf{z}_{\boldsymbol{a} \boldsymbol{b} \boldsymbol{l}}$.

The ablation experiment with both parameter sets leads to the following relationship between the roughness depth $S_{z}$ and the ablation depth $z_{a b l}$ :

$$
S_{Z}\left(z_{a b l}\right)=\left(S_{Z, I S U}-S_{Z, \infty}\right) e^{-g z_{a b l}}+S_{Z, \infty}
$$

The factor $g$ is introduced and defined as the smoothing constant of the function. In its mathematic meaning $g$ is comparable with the damping constant in damped oscillations or the decay constant in radioactive decay. The smoothing constant $g$ is given in the unit $\mu \mathrm{m}^{-1}$ and only assumes values $>0$. It represents the quantification of the smoothing ability of a parameter set like described in section 3.1. Accordingly, a high smoothing constant represents a great smoothing potential. The exponential decrease of the $S_{z}$ curve would be steeper in the case of a great smoothing constant, whereas a low smoothing constant is represented in a slower decline.

With great accuracy, the mathematical model corresponds to the measured data points of both experiments. The approximation by the model for parameter set 1 is shown in Fig. 12. If the smoothing constant $g$ can only be determined experimentally or if it can be calculated using other process variables or estimated with a simulation cannot be answered in the context of this paper and requires further investigations.

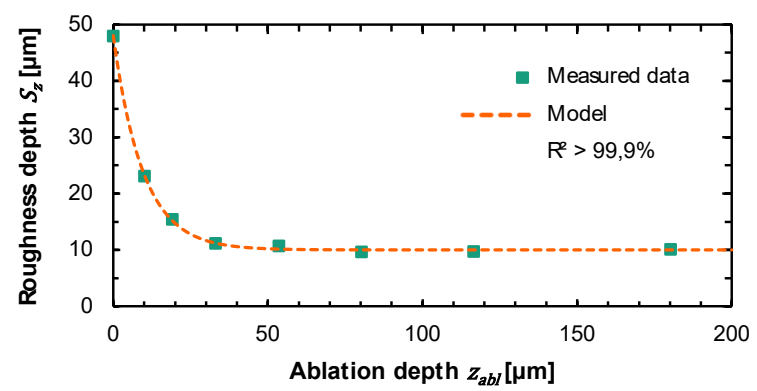

Fig. 12 Mathematical model for the evolution of the roughness

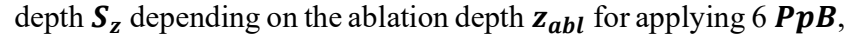
$\boldsymbol{F}_{\mathbf{0}}=1.5 \mathrm{~J} / \mathrm{cm}^{2}, \boldsymbol{S}_{\boldsymbol{Z}, \boldsymbol{A} \boldsymbol{O}}=48 \mu \mathrm{m}$ and $\boldsymbol{S}_{\boldsymbol{Z}, \infty}=10 \mu \mathrm{m}$. The smoothing constant $\boldsymbol{g}$ can be determined to $\boldsymbol{g}=0.1043 \mu \mathrm{m}^{-1}$ with a model accuracy of $\mathrm{R}^{2}>99.9 \%$.

\section{Photonic process chain with ns and ps ablation}

In the previous sections, the foundations were laid for a sequentially photonic process chain to combine productivity and quality. In the following a process example for sequential ns/ps ablation will be analyzed with respect to time savings and the development of surface quality. The example shows a combined ablation process, which consists of ns coarse ablation and ps fine ablation. The development of the respective $S_{a}$ spectrum is shown in Fig. 13 .

For the ns coarse ablation, productive parameters are selected as far as possible, where quality is of secondary importance. This goal is achieved by the parameter for producing ISU ns1 (cf. Fig. 3). The ablation rate is $d V / d t=17.4 \mathrm{~mm}^{3} / \mathrm{min}$ while achieving a surface roughness with $S_{a}=1.64 \mu \mathrm{m} / S_{z}=19.9 \mu \mathrm{m}$.

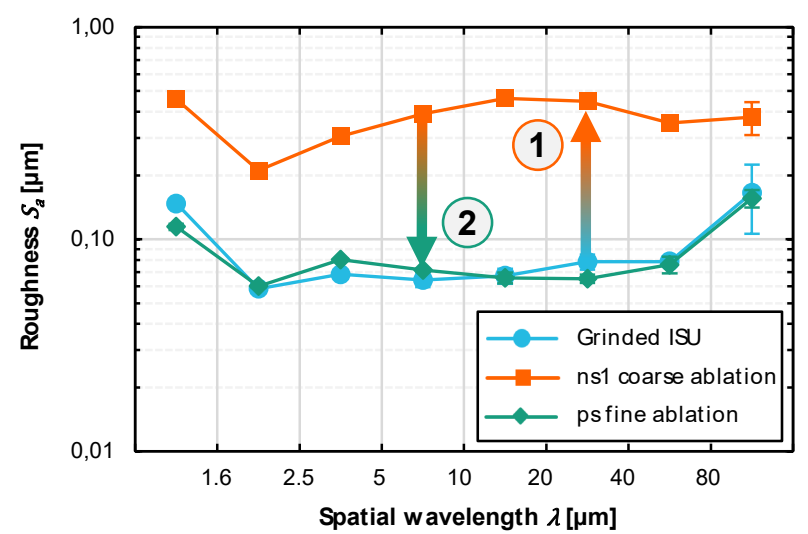

Fig. 13 Development of the $\boldsymbol{S}_{\boldsymbol{a}}$ spectrum over the spatial wavelength for three surfaces with different conditions. First the grinded initial surface (blue, $\boldsymbol{S}_{\boldsymbol{a}}=0.38 \mu \mathrm{m} / \boldsymbol{S}_{\boldsymbol{z}}=7.6 \mu \mathrm{m}$ ) is processed by ns coarse ablation (orange, $\boldsymbol{S}_{\boldsymbol{a}}=1.64 \mu \mathrm{m} / \boldsymbol{S}_{\boldsymbol{z}}=19.9 \mu \mathrm{m}$ ) before second applying ps fine ablation (green, $\boldsymbol{S}_{\boldsymbol{a}}=0.35 \mu \mathrm{m}$ $\left.\boldsymbol{S}_{\mathbf{z}}=8.8 \mu \mathrm{m}\right)$. The parameter for ns coarse ablation is equal to the ns parameter producing ISU ns 1 (cf. Fig. 3). The line distance $\boldsymbol{L} \boldsymbol{D}$ is about $24 \mu \mathrm{m}$ that explains the local maximum roughness at this

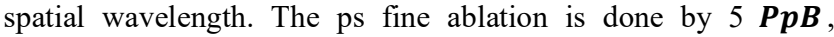
$\boldsymbol{F}_{\mathbf{0}}=1.5 \mathrm{~J} / \mathrm{cm}^{2}, \quad \boldsymbol{N}=74$ resulting in an ablation depth $z_{a b l}=42.2 \mu \mathrm{m}$.

For the subsequent ps fine ablation, the focus is on the best possible quality. A parameter set that achieves excellent quality on both coarsely ablated and grinded initial surfaces is $P p B=5$ with $F_{0}=1.5 \mathrm{~J} / \mathrm{cm}^{2}$. After the ps fine ablation, the global roughness is about $S_{a}=0.35 \pm 0.02 \mu \mathrm{m}$. The 
quality of the two-stage ablated surface obtains a comparable surface roughness like the original grinded ISU with $S_{a}=0.38 \pm 0.02 \mu \mathrm{m}$.

Fig. 14 shows a diagram with the ablated volume vs. the process time for the sequentially processes applying the same processing parameter like mentioned above. The coarse ablation removes $80 \%$ and the fine ablation $20 \%$ of the total ablation volume of $100 \mathrm{~mm}^{3}$. The large time saving as a result of the sequential ablation process strategy is obvious. Applying a first productive ns coarse ablation followed by a fine ps ablation $75 \%$ of the process time is saved compared to a single ps ablation while retaining the surface quality (cf. Fig. 13). A further significant shortening of the process time while maintaining the quality would be possible by increasing the proportion of coarse ablation. The limitation is to keep a minimum ablation depth for ps fine ablation to be able to achieve the desired surface quality (cf. section 3.3).

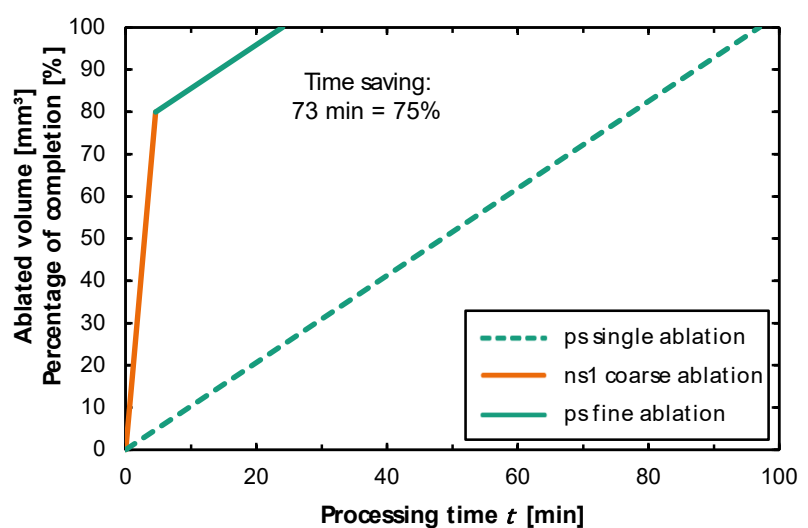

Fig. 14 Time saving of the two-stage sequentially ns/ps ablation process compared to the single ps ablation. The ns coarse ablation is represented by the parameter producing ISU ns1 (cf. Fig. 3) with an ablation rate of $17.4 \mathrm{~mm}^{3} / \mathrm{min}$. The ps fine ablation (single and two-stage) is done by $5 \boldsymbol{P p} \boldsymbol{B}, \boldsymbol{F}_{\mathbf{0}}=1.5 \mathrm{~J} / \mathrm{cm}^{2}$ gaining an ablation rate of $1.1 \mathrm{~mm}^{3} / \mathrm{min}$.

The required minimum removal depth of the ps fine ablation could be significantly reduced by an intermediate laser based smoothing process. This could not only increase the portion of the coarse ablation, but also extend the field of possible ps parameter. A parameter set with low smoothing ability that would induce rough microstructures without intermediate smoothing could be used, since the roughness depth of the ISU would be reduced below the threshold roughness depth of the parameter set $\left(S_{z, I S U}<S_{z, t h r}\right)$. Laser based smoothing processes without material removal as an additional process step in the sequentially photonic process chain should be investigated in further examinations.

\section{Conclusion}

Many investigations deal with the laser inherent parameters like pulse duration, repetition frequency and scan speed to describe their influence on the machining quality. However, the final machining quality is also determined by the initial surface morphology. The knowledge about the influence of the respective surface morphology on the subsequent process step is of decisive importance.

Ultrashort pulsed burst processing reveals the potential of smoothing the surface within the ablation process. With the exactly right amount of heat accumulation, a smooth surface with a homogeneous melt film is achieved. This circumstances can be used for a sequentially combined process using productive ns ablation supplemented by high quality ps ablation.

The findings presented in the underlying paper show the dependency of the accessible surface quality on the initial surface roughness (cf. Fig. 5 and Fig. 6). By utilizing the smoothing potential of ultrashort pulsed burst processing the initial by ns coarse ablation achieved rough surfaces can be controlled (cf. Fig. 7). An initially rough surface (ISU ns5) with a roughness depth of $S_{z}=44.7 \mu \mathrm{m}$ (respectively $S_{a}=4.71 \mu \mathrm{m}$ ) was able to be smoothed by a factor of 4 while ablating with $8 P p B$ and $F_{0}=1.0 \mathrm{~J} / \mathrm{cm}^{2}$ to $S_{z}=10.6 \mu \mathrm{m}$ (respectively $S_{a}=0.66 \mu \mathrm{m}$ ). The examinations observed that in ultrashort pulsed laser ablation with bursts each process parameter combination can be characterized by a smoothing ability. The smoothing mechanism is based on the combination of two process variants. On the one hand, the smoothing is carried out by means of ablation, in which the profile peaks are removed more than the rest of the surface. On the other hand, parallel to the ablation process the material is remelted. The smoothing potential is linked to a specific threshold roughness depth $S_{z, t h r}$. If the initial roughness depth of the ISU $S_{Z, I S U}$ is below this threshold $\left(S_{Z, I S U}<S_{Z, t h r}\right)$, the roughness of the ISU can still be positively influenced by ps ablation. Additionally the final roughness depth depends on the ablation depth $z_{a b l}$ that is asymptotic saturated to $S_{z, \infty}$ by rising ablation depth. Based on the observations a mathematical approach is developed that introduced $g\left[\mu^{-1}\right]$ as a smoothing constant connected to each ps ablation parameter set. The developed model fits very well to the measured data with $\mathrm{R}^{2}>99.9 \%$. With the knowledge of the smoothing ability of a ps parameter that is linked to the parameter specific saturation roughness $S_{z, \infty}$ the necessary ablation depth can be calculated to smooth a coarse ablated surface. With a right process parameter setting (allocated to the 2 nd heat accumulation regime) the final surface roughness produced by the subsequent ps ablation is independent on the initial surface morphology.

On all laser-pretreated initial surfaces a higher productivity of the ps ablation can be observed in comparison to reference ablation on the grinded initial surface. This observation must not be taken as a general result of the advanced incubation effect. The main reason for the higher ablation rate on the laser pretreated surfaces seems to be the measured higher absorption coefficient. There is a correlation to the greater roughness depth of the laser-ablated initial surfaces.

The presented work in the underlying paper presents the potential of a complete digital sequentially photonic process chain. The laser source is a universal tool that reveals many options for material processing. Currently in the context of surface structuring only one parameter is used that is limited to focus either on productivity or on quality. The results shown here expose that with a simple two-step process applying ns and ps pulses the processing time can be reduced by up to $75 \%$ while achieving quality as good as with single ps ablation $\left(S_{a}=0.36 \mu \mathrm{m}\right)$.

Further investigations should apply to the development of an intermediate laser based smoothing process. Polishing 
the coarse ablated rough surface by remelting without ablation as an additional process step of the sequentially photonic process chain will increase the application possibilities for ps ablation processes. In addition examinations should be done on sequentially surface structuring using only an ultrafast laser source. For this purpose, productive process parameters using especially pulse bursts would be selected for coarse ablation, which would predominantly induce thermal ablation. A further modulation of the process parameters finally ensures a selective adjustment of the surface, so that an evaporation-dominated fine ablation for the generation of microstructures can take place.

\section{Acknowledgments}

This work is supported by the German Federal Ministry of Education and Research (BMBF) under the grant number 02P14A145 (eVerest).

\section{References}

[1] G. Ryk and I. Etsion: Wear, 261, (2006) 792.

[2] J. Schneider, D. Braun, and C. Greiner: Lubricants, 5, (2017) 1 .

[3] A. Dunn, K. L. Wlodarczyk, J. V. Carstensen, E. B. Hansen, J. Gabzdyl, P. M. Harrison, J. D. Shephard, and D. P. Hand: Appl. Surf. Sci., 357, (2015) 2313.

[4] A. Dohrn and A. Gillner: Application Center. Laser Structuring for Tool and Mold Construction, $<$ https://www.ilt.fraunhofer.de/content/dam/ilt/en/documents/product_and_services/laser_material_processing/B_Application_Center_Laser_Structuring_for_Tool_and_Mold_Construction 2015.pdf $>$.

[5] C. Zhou, G. Li, C. Li, Z. Zhang, Y. Zhang, S. Wu, Y. $\mathrm{Hu}$, W. Zhu, J. Li, J. Chu, Z. Hu, D. Wu, and L. Yu: Appl. Phys. Lett., 111, (2017) 141607.

[6] C.-W. Wang, Y.-Y. Dang, Y.-H. Su, J.-C. Ni, C.-C. Zhang, J.-W. Li, Y.-L. Hu, J.-R. Chu, W.-H. Huang,
D. Wu, and X.-T. Tao: IEEE Photonics J., 9, (2017) 1 .

[7] A. Brenner, F. Kurzidim, C. Fornaroli, and A. Gillner: J. Laser Appl., 30, (2018) 32509.

[8] B. N. Chichkov, C. Momma, S. Nolte, F. Alvensleben, and A. Tünnermann: Appl. Phys. A, 63, (1996) 109.

[9] Y. Zhu, J. Fu, C. Zheng, and Z. Ji: Opt. \& Laser Tech., 83, (2016) 21.

[10] K.-H. Leitz, B. Redlingshöfer, Y. Reg, A. Otto, and M. Schmidt: Phys. Proc., 12, (2011) 230.

[11] B. Neuenschwander, B. Jaeggi, T. Kramer, and S. Remund: ICALEO, (2016) 1.

[12] A. Brenner, B. Bornschlegel, and J. Finger: J. Laser Micro/Nanoengin., 14, (2019) 100.

[13] J.-T. Finger: Puls-zu-Puls-Wechselwirkungen beim Ultrakurzpuls-Laserabtrag mit hohen Repetitionsraten, (Apprimus Verlag, Aachen, 2017).

[14] B. Neuenschwander, G. F. Bucher, C. Nussbaum, B. Joss, M. Muralt, U. W. Hunziker, and P. Schuetz: SPIE LASE. Proc. SPIE, 7584, (2010) 75840R.

[15] B. Lauer, B. Jäggi, and B. Neuenschwander: Phys. Proc., 56, (2014) 963.

[16] B. Neuenschwander, B. Jaeggi, M. Schmid, and G. Hennig: Phys. Proc., 56, (2014) 1047.

[17] J. Sotrop, J. Winter, S. Rapp, and H. P. Huber: SPIE New., http://spie.org/news/6004-understanding-laserablation-efficiency?SSO=1, (2015) 1.

[18] B. Neuenschwander, B. Jaeggi, M. Schmid, A. Dommann, A. Neels, T. Bandi, and G. Hennig: SPIE LASE. Proc. SPIE, 8607, (2013) 86070D.

[19] A. Y. Vorobyev and C. Guo: Appl. Phys. Lett., 86, (2005) 11916.

[20] J. Byskov-Nielsen, J.-M. Savolainen, M. S. Christensen, and P. Balling: Appl. Phys. A, 101, (2010) 97.

(Received: May 27, 2019, Accepted: October 6, 2019) 